\title{
Influence des sols contaminés en cuivre sur le développement de deux variétés (locale et améliorée) de légumes dans la région de Lubumbashi (RD. Congo).
}

\author{
Mpundu Mubemba Mulambi Michel ${ }^{102}$, Mununga Katebe Félicien ${ }^{1 *}$, Kaumbu Kyalamakasa Jean-Marc ${ }^{1}$, \\ Mwilambwe Kapoma Xavier ${ }^{1}$, Maloba Kazembe Jean-Pierre ${ }^{3}$, Banza llunga Meschack ${ }^{3}$, Mukunto \\ Kimonge Ismaël ${ }^{4}$. \\ ${ }^{1}$ Département de Phytotechnie, Faculté des Sciences Agronomiques, Université de Lubumbashi, BP 1825 \\ Lubumbashi, RD Congo ; \\ 2École d'Hôtellerie et Tourisme. BP 1825, Université de Lubumbashi, BP 1825 Lubumbashi, RD Congo \\ ${ }^{3}$ Centre de Recherche Agro-alimentaire(CRAA) Lubumbashi, RD Congo. \\ 4Département d'Agro-vétérinaire, Institut Supérieur Pédagogique, BP 1798 Lubumbashi, RD Congo \\ "Auteur correspondant : munungakatebe@gmail.com , +243815778141.
}

Original submitted in on $6^{\text {th }}$ March 2017. Published online at www.m.elewa.org on 31st July 2017 https://dx.doi.org/10.4314/jab/v115i1.1

\section{RESUME}

Objectifs : Dans l'ex province du Katanga l'agriculture vivrière est majoritairement traditionnelle. Alors que ces sols sont contaminés par les etms provenant des activités industrielles ont pour conséquences l'augmentation des diverses substances riches en etms (zinc, cobalt, plomb, arsenic, cuivre, cadmium). Cette étude avait pour objectif d'évaluer le comportement de la culture de carotte et de l'aubergine africaine installées sur les sols contaminés et de déterminer le risque potentiel de la contamination de la chaine alimentaire par les ETMs dans la ville de Lubumbashi.

Méthodologie et résultats : quatre types de sol ont été récoltés en vue d'y installer les deux cultures (Carotte et aubergine africaine). L'expérimentation a été conduite selon un dispositif factoriel $4 * 2$, ainsi le facteur principal était constitué de deux variantes de carotte et d'aubergine (variété améliorée et variété locale) et le facteur secondaire était constitué de 4 variantes de sol dont (le sol de Kipushi, sol de Pengapenga, sol de Luwowoshi (Ruashi) ainsi que le sol de la ferme Kassapa). Les résultats obtenus ont révélés que les sols des jardins potagers de la ville de Lubumbashi, ainsi que les biomasses de carotte installées sur ces substrats ont présentent des concentrations très élevées par rapport à la norme établie, contrairement aux biomasses d'aubergine qui n'ont pas présentées de risque de contamination quelque soit la contamination des substrats. Conclusion et application des résultats : Les variétés améliorées de carotte et d'aubergine provenant de la France ce sont bien comportées comparativement aux variétés locales utilisées couramment par les agriculteurs de la ville de Lubumbashi, nous conseillerons aux agriculteurs d'utiliser ces variétés mises à leur disposition pour éviter les risque de contamination de la chaine alimentaire et dans les prochaines études qu'une expérimentation in situ soit menée en utilisant les mêmes variétés.

Mots clés : Variété, sol contaminé, carotte, aubergine africaine, chaine alimentaire. 


\begin{abstract}
Objectives : In the former province of Katanga, subsistence agriculture is predominantly traditional. While these soils are contaminated by industrial effluent, the result is an increase in the number of substances rich in etms (zinc, cobalt, lead, arsenic, copper, cadmium). The objective of this study was to assess the behavior of African carrot and eggplant culture on contaminated soils and to determine the potential risk of food chain contamination by the MTEs in Lubumbashi City.

Methodology and results : Four soil types were harvested to install the two crops (African carrot and aubergine). The experiment was carried out in accordance with a factor 4 * 2 apparatus, thus the main factor consisted of two variants of carrot and aubergine (improved variety and local variety) and the secondary factor consisted of 4 soil variants including Kipushi soil, Pengapenga soil, Luwowoshi soil (Ruashi), and Kassapa farm soil. The results showed that the soils of the vegetable gardens of the city of Lubumbashi, as well as the carrot biomasses installed on these soil, showed very high concentrations compared to the established norm, whereas the eggplant biomasses that did not Contamination, regardless of the contamination of the substrates. Conclusion and application of results: Improved varieties of carrots and eggplants from France are well behaved compared to local varieties commonly used by farmers in the city of Lubumbashi, we will advise farmers to use these varieties Provision to avoid the risk of contamination of the food chain and in the next studies that an in situ experimentation is carried out using the same varieties.
\end{abstract}

Keywords: Variety, contaminated soil, carrot, African eggplant, food chain.

\section{INTRODUCTION}

L'agriculture vivrière est généralement traditionnelle dans l'ex province du Katanga (FAO 1998), en Afrique nombreuses familles pratiquent des cultures maraichères en milieu urbain, c'est ainsi que cette activité contribue sensiblement à la fourniture des légumes frais (Cissé et Moustier 1999 ; Easton et Hilhorst, 2003 ; FAO 2008 ; Mpundu 2010). Alors la population mondiale va augmenter de plus de 3 milliards d'individus d'ici 2030, mais cette croissance démographique va nécessiter de doubler la production agricole, pour arriver à subvenir à leurs besoins Mougeot et Moustier, 2004 ; Mougeot, 2006). Cette augmentation démographique et l'industrialisation de la province génèrent les rejets pouvant contaminer l'environnement (Kitobo 2009), et qui troublent le fonctionnement des écosystèmes et entrainent des troubles sur les êtres vivants (Mpundu, 2010). Par ailleurs, des indices de contaminations et d'une acidification importante des sols par les retombées des poussières métallifères ont été constatés autour des usines de traitement de minerais et jusqu'à plusieurs kilomètres de distance (Mpundu et al., 2013). Cette activité minière est la cause principale des plusieurs atteintes à l'environnement. En effet, depuis l'extraction du minerai des gisements par l'établissement des carrières jusqu'à celle du métal spécifique recherché à l'industrie, l'activité minière rejette les déchets et effluents (gazeux, liquide, solides) car le métal recherché n'est qu'une infirme fraction du volume de la roche déplacée (Imper et al., 1991). Les sols agricoles n'ont pas été épargnés par cette atteinte, ce qui y provoque la baisse de production végétale tant du point de vue qualitatif que quantitatif. Toutes fois nombreuses usines hydro-métallurgiques et fonderies à Lubumbashi rejettent les effluents contenant de sous-produits riches en zinc, plomb, arsenic, cadmium ou les composés soufrés à cause de l'extraction du cuivre et du cobalt (Ngoy et al., 2006). Cependant ces effluents rejetés ont un impact néfaste sur l'environnement: l'air, l'eau et sol accumulent des contaminants, leurs fonctionnements et propriétés sont perturbés, toute fois des troubles sur les êtres vivants et leur environnement sont constaté (Bruneau, 1983 ; Tembo et al., 2005 ; Ngoy et al., 2006). Les études effectuées par Mattson \& Guidotti, 1980 aux états unis et au canada, ont montrées qu'une augmentation des maladies respiratoires a été observée chez les femmes âgées de 37 à 75 ans habitant aux alentours des installations et fonderies électriques de cuivre (Mattson \& Guidotti, 1980). Des 
mêmes, dans les tissus et fruits environnant, des teneurs élevées en métaux lourds ont été trouvées. Une multitude d'exemples notamment la catastrophe de minamata qui avait affecté une population des pêcheurs après consommation d'une forte proportion de chair des poissons contenant des fortes concentrations de méthylmercure. Cependant l'intoxication massive a eu comme conséquence le dénombrement de 121 cas d'intoxication à symptomatologie nerveuse dont 54 décès entre 1953 et 1971 (Buchet et al., 1983). Aux états unis il se produit une intoxication aigue au cadmium par voie alimentaire après une consommation de jus des fruits souillé par 200mg de Cd par litre En 1944 et en 1946 en Algérie 946 cas d'intoxication furent observés dans une cantine après ingestion de vin ayant séjourné pendant 4 à 6 heures dans les bacs en métal cadmié (Boudeme, 2000). Dans le HautKatanga, les eaux de lavage des minerais, les effluents des usines de traitement et d'enrichissement rejetés sans traitement préalable dans les rivières, et les eaux de ruissellement des terrils contiennent en solution, et dans leurs sédiments des métaux lourds, ainsi que les résidus acides et des différents produits chimiques intervenant soit, dans les processus d'extraction et de séparation, soit dans les processus de raffinage (Mpundu, 2010). Ces polluants solubles et même toxiques détruisent les biotopes aquatiques en aval sur des distances importantes, sont métabolisés dans l'ensemble de la chaîne alimentaire et, de ce fait, présentent des risques pour la santé des populations riveraines (Vande Weghe et al., 2005). Par ailleurs, dans la région de Lubumbashi en particulier, il a été démontré que les émissions de $\mathrm{SO}_{2}$ par la cheminée du four de l'usine d'extraction et de transformation du cuivre de la Gécamines étaient garants de pluies acides (Mbenza et al., 1989), nocives pour les êtres vivants dont les végétaux. En outre il n'a été constaté que le secteur établi sous les vents alizés du Sud-est présente des sols riches en cuivre, et autres métaux lourds, par les retombées de poussières métallifères émanant de l'usine depuis plus d'un demi-siècle. C'est pourquoi cette situation avait favorisée le remplacement de la forêt claire originelle de Miombo, par une steppe courte au sein de laquelle on retrouve beaucoup d'espèces de la flore cupricole (Malaisse, 1997) ainsi que de vastes zones de sol dénudé. Entretemps, le sol resté nu devient l'objet d'une érosion hydrique et éolienne soutenue. A cela s'ajoute une pression anthropique croissante accentuant le phénomène d'érosion et le risque de transfert des ETM dans la chaîne alimentaire.

Les études menées par Shutcha (2010) et Mpundu (2010) montre que l'amendement organique ne réduit pas suffisamment la mobilité et la biodisponibilité des ETMS sur le sol contaminé et que son efficacité est significative à des plus grandes doses mais son importance reste toujours dans l'amélioration de la richesse spécifique et le recouvrement que l'amendement calcaire dont ce dernier à des effets négatifs pour les prochaines cultures. Alors la matière organique utilisée doit être riche en éléments nutritifs et doit être à une dose plus élevée. Bien que cela l'amendement organique reste meilleur car il est facile à appliquer et moins couteux mais toutes fois, il peut être combiné à la chaux pour les bonnes efficacités et combinés aux autres engrais fertilisants dans le cas de sols plus pauvres en éléments nutritifs de plantes dont la quantité apportée avec la matière organique est insuffisante. Les matières organiques peuvent agir en tant que ligands sur les métaux, modifiant leur spéciation, leur dynamique ou leur impact en tant que polluants. L'apport d'amendements organiques sur des sols contaminés peuvent non seulement relever le pH mais, aussi entraîner une immobilisation partielle des polluants (Clémente et al., 2006). Les matières organiques interviennent dans la rétention des métaux par leurs groupements carboxyles R-COOH, phénol R-OH, amines R-NH2 et thiols R-SH. Les effets de la matière organique sur la mobilité incluent l'adsorption des ions échangeables et la chélation. La matière organique est un adsorbant qui diminue ou modifie l'activité et le transport des ETM dans le sol (Liu \&Wang Z.J., 1996 ; Kabala \& Szersezn, 2002 ; Plusieurs études ont déjà montré les effets positifs de la diminution de la mobilité des ETM par les apports des amendements organiques dans les sols (Zhang et al., 2003 ; Hua et al., 1998 ; Bride, 1989). Cependant la composition des amendements organiques est un 
facteur très important. On peut distinguer dans la famille des composés organiques :

- les matières organiques humifiées composées d'acides fulviques, d'acides humiques et d'humine, ainsi que

- de ligands organiques de faible poids moléculaire, essentiellement issus de l'activité biologique du sol (exsudats racinaires) (Duchaufour, 2001 ; Bataillard, 2002).

La matière organique soluble (acides fulviques), favorise la mobilité et l'activité biologique des ETM. Par contre, les composés organiques à molécules élevées (humines) adsorbent et diminuent la mobilité et l'activité biologique des ETM (Deneux- Mustin et al., 2005 ; Ruttens et al., (2006), a observé que l'addition du compost à un sol contaminé en France, a réduit légèrement la phytotoxicité de 3,5 à 2,8 et l'accumulation du métal dans les plants de la fève où les concentrations de $\mathrm{Zn}, \mathrm{Cd}$ et $\mathrm{Pb}$ ont diminué respectivement de 623 à 135, de 6 à 1 et de 11 à 6 mg par kg de poids sec. Ruttens et al., (2006) a également montré que, l'application du compost dans un sol riche en métaux lourds en France, a induit une diminution de la teneur en métaux lourds de l'ordre de $99 \%$ de Zn, $99 \%$ de Cd, $97 \%$ de Cu et $98 \%$ de $\mathrm{Pb}$. Les etms présent dans le sol posent

\section{MATERIELS ET METHODES :}

La ville de Lubumbashi, chef lieu de la province du Haut-Katanga est caractérisée par les coordonnées géographiques suivantes: $1276 \mathrm{~m}$ d'altitude dans le carré formé par les latitudes $11^{\circ} 36^{\prime} 31.0^{\prime \prime}$ Sud et les longitudes $27^{\circ} 18^{\prime} 36.4^{\prime \prime}$ Est. Le climat de la ville de Lubumbashi est du type Cw6 selon la classification de Koppën (FAO, 2005). Ce climat est du type tropical est déterminé par l'alternance de deux saisons, bien marquées. Premièrement, la saison des pluies qui va de novembre à mars, deuxièmement, la saison sèche qui débute de mai à septembre, mais Avril et Octobre sont considérés comme mois de transition. Lubumbashi et ses environs sont caractérisés par une température moyenne annuelle de l'ordre de $20^{\circ} \mathrm{C}$ alors que les précipitations moyennes annuelles sont estimées près de $1200 \mathrm{~mm}$. Une couverture végétale naturelle de la ville de Lubumbashi et deux sérieux problèmes pour l'homme notamment dans le domaine agricole dont la plupart des etms contenus dans le sol sont transférer vers les plantes (Mpundu, 2010 ; Mpundu et al., 2013, puis dans le domaine de santé publique, la contamination de la chaine alimentaire par les etms entraine un phénomène de bioconcentration des etms à leur passage dans le maillon trophique supérieur (Gonzales et al., 2008 ; Katemo, 2009 ; McLean et al., 2009). Cependant différents procédés de réhabilitation des sols existent déjà, mais la phytostabilisation est une des méthodes la moins couteuse et la plus adaptée (Mpundu, 2010 ; Ngoy, 2010). C'est ainsi que l'application des amendements dans la phytostabilisation permet d'améliorer les propriétés physico-chimiques du sol ayant des teneurs d'etms très élevées (Mulaji, 2010), alors que l'application de la chaux au sol pollués réduit sensiblement la biodisponibilité des etms dans le sol (Mpundu, 2010; Ngoy, 2010). Cette a été menée en vue d'évaluer le comportement de la culture de carotte et de l'aubergine africaine installées sur les sols contaminés et déterminer le potentiel risque de la contamination de la chaine alimentaire par les ETMs dans la ville de Lubumbashi.

ses environs est une forêt claire du type Miombo. Autour de la ville de Lubumbashi cependant, cette forêt est en train d'être remplacée par les savanes (Munyemba, 2010 ; Cabala, 2012). La couverture pédologique de la ville de Lubumbashi est du type ferralitique avec un pH à l'eau oscillant autour de 5,2 (Kasongo et al., 2013).

Matériel biologique : Notre étude avait porté sur deux variétés de (carotte et d'aubergine) génétiquement modifiées provenant de la France et deux variétés locales de (carotte et d'aubergine) couramment utilisées par les maraichers dans la ville de Lubumbashi. Les semences pour la variété améliorée ont été achetées au marché Kenya dans la boutique AGRISEM spécialisée pour la vente des semences génétiquement modifié, alors que les semences locales ont été achetées au marché Mzee au près des vendeurs ordinaires. 

variétés (locale et améliorée) de légumes dans la région de Lubumbashi (RD. Congo).

Tableau1 : Concentration en cuivre dans les différents sols des jardins potagers de la région de Lubumbashi.

\begin{tabular}{|l|c|c|c|}
\hline Traitement & Cuivre en ppm & Norme en ppm & Référence \\
\hline Pengapenga & $332 \mathrm{ppm}$ & $100 \mathrm{ppm}$ & Coppenet et al., 1993; Chevery, 1994 \\
\hline Ruashi & $2070 \mathrm{ppm}$ & $100 \mathrm{ppm}$ & \\
\hline Kipushi & N.D & $100 \mathrm{ppm}$ & \\
\hline Kassapa & $115 \mathrm{ppm}$ & $100 \mathrm{ppm}$ & \\
\hline
\end{tabular}

Légende : N.D : Non déterminé ; ppm : parti par million

Partant du tableau 1 , les résultats de laboratoire montrent que de tous les quatre types de sol utilisés, le sol de Rwashi avait présenté de teneurs 20 fois supérieures à la norme, alors que le sol de Pengapenga avait présenté de teneurs 3 fois supérieures à la norme, mais le sol de Kassapa considéré comme témoin avait excéder légèrement la norme ; contrairement au sol de Kipushi qui n'avait pas présenté de traces de contamination cuprique Méthodes : Deux expérimentations ont été conduites dans les sachets en polyéthylènes, installées au champ expérimental de la faculté des sciences agronomiques de l'université de Lubumbashi, suivant un dispositif factoriel $4 * 2$, le premier facteur constitue les deux variantes d'aubergine et de carotte (variété améliorée et celle locale) et le second facteur est constitué par les quatre variantes de sol dont celui (de Kipushi, de Pengapenga, de Ruashi ainsi que celui de la ferme Kassapa).

Analyse chimique: Les plantes de carotte et d'aubergine récoltées ont été lavés, puis placées dans les enveloppes et ces dernières étaient posées dans l'étuve à une température de $40^{\circ} \mathrm{C}$ pendant 24 heures pour leur séchage, par suite ces échantillons ont été broyés et mises dans des petites boites. Les opérations de laboratoire ont consistés à émietter les grosses particules de sol après séchage, le triage et le pesage. C'est ainsi

\section{RESULTATS}

Levée et survie de plantes de Solanum macrocarpon: Les résultats de l'analyse de la variance révèlent des différences significatives entre les traitements $(p=0,000)$ pour ce qui est de la levée à 7 jours ainsi que de la survie à toutes les dates d'observations. Par rapport à l'utilisation des types de sols, le meilleur taux de levée a été obtenu avec le sol de Kipushi (85\%), alors que le sol de la ferme Kassapa et de la Ruashi ont présenté des taux de levée intermédiaires, et le plus faible taux de levée a été obtenu avec les sols de Pengapenga. L'analyse de la variance ne révèle pas des différences significatives $(p=0.81)$, c'est-à-dire que l'utilisation de la variété n'a pas eu d'effet sur la levée à 7 jours ; quant à l'utilisation des variétés les meilleurs taux de levée ont été obtenus avec la variété locale installé sur le sol de Ruashi que les échantillons des sols ont été misent dans des petites boites portant les indications se rapportant aux sites où ont été échantillonnés les sols. La détermination des éléments traces métalliques dans les végétaux tout comme dans les sols a été réalisé à l'aide d'un appareil à fluorescence $X$ de marque OLYMPUS. Cette analyse chimique avait consisté à doser les différents éléments chimiques. Ce dosage s'est effectué par spectrométrie de fluorescence $X$. Toutefois, la fluorescence $X$ est une méthode analytique non-destructive des échantillons dont le principe de base est l'irradiation d'un échantillon par des rayons $X$. une fois qu'un échantillon absorbe les rayons incidents, celui-ci émet une fluorescence ou des radiations situées dans le domaine des rayons $X$. Ces analyses ont été effectuées au laboratoire Agropédologique de la faculté des sciences agronomiques(FSA).

Analyse statistique : Les analyses statistiques ont été réalisées à l'aide du logiciel Minitab 16 pour voir l'effet des types de sol sur les paramètres végétatifs notamment (le nombre de feuilles, le taux de survie, la hauteur des plantes) de la carotte. Les analyses de la variance pour les paramètres végétatifs ont été réalisées à l'aide des logiciels R et Minitab.16. Le test de Tukey a été utilisé envue de comparer les différentes moyennes.

et Kipushi. Par rapport à l'utilisation des types de sols, le meilleur taux de survie a été obtenu avec le sol de Kassapa, Kipushi et Ruashi à 30 jours, alors que le sol de Pengapenga avait présenté un taux de survie faible, paradoxalement à 45 et 60 jours seuls le sol de Kassapa et Kipushi avait un taux de survie élevé contrairement aux sol de Pengapenga et Ruashi ont donnés une survie nulle. L'analyse de la variance révèle des différences significatives entre les traitements $(p=0.004)$ à toutes les dates d'observation, sauf à 45 jours, c'est-à-dire la variété a influencer significativement la survie à 30,60 et 90 jours après semis ; le meilleur taux de survie a été obtenu avec le sol de Ferme Kassapa; les sols de Kipushi et de Ruashi, alors que le faible taux de levée a été obtenu avec le sol du quartier Pengapenga. Toutefois 

variétés (locale et améliorée) de légumes dans la région de Lubumbashi (RD. Congo).

l'interaction entre traitement et la variété a eu des effets significatifs à toutes les dates d'observations sauf à 45 jours où l'effet était non significatif entre les traitements. Nombre des feuilles : Dans le tableau 3, par rapport à l'utilisation des types de sols, le meilleur sol, ayant donné un bon nombre des feuille a été obtenu avec le sol de Kipushi suivit de Kassapa à toute les différentes dates d'observations. Cependant, les sol de Ruashi et le sol de Pengapenga ont présentés un taux faible en nombre des feuilles, paradoxalement à 30, 45, 60 et 90 jours ; seuls le sol de Kipushi et Kassapa avait présenté des taux élevé contrairement aux sol de Pengapenga qui a donné un faible taux en nombre des feuilles à 30 jours seulement, et sur le sol de Ruashi où la quantité des feuilles sur les plantes n'ont pu évoluer depuis 30 ème jusqu'au $45^{\mathrm{eme}}$ jour, alors qu'au 60 et 90 éme jour, ils ont donné une valeur nulle en nombre des feuilles.

Tableau 2 : Taux de levée à 7 jours et survie des plantes de Solanum macrocarpon 90 jours après semis

\begin{tabular}{|l|l|l|l|l|l|l|}
\hline Variété & Traitement & TL7 & TS30 & TS 45 & TS60 & TS90 \\
\hline \multirow{4}{*}{$\begin{array}{l}\text { Variété } \\
\text { Améliorée(Afrisem) }\end{array}$} & S.KAS & $71.88 \pm 10.83$ & $100 \pm 0$ & $100 \pm 0$ & $100 \pm 0$ & $100 \pm 0$ \\
\cline { 2 - 7 } & S.KIP & $84.38 \pm 14.88$ & $100 \pm 0$ & $100 \pm 0$ & $100 \pm 0$ & $100 \pm 0$ \\
\cline { 2 - 7 } & S.P & $29.69 \pm 10.67$ & $100 \pm 0$ & $0 \pm 0$ & $0 \pm 0$ & $0 \pm 0$ \\
\cline { 2 - 7 } & S.RWA & $71.88 \pm 71.88$ & $100 \pm 0$ & $100 \pm 0$ & $0 \pm 0$ & $0 \pm 0$ \\
\hline \multirow{5}{*}{ Moyenne } & S.KAS & $64.45 \pm 23.58$ & $100 \pm 0.00$ & $75 \pm 44.72$ & $50 \pm 51.6$ & $50 \pm 51.64$ \\
\cline { 2 - 7 } & S.KIP & $85.94 \pm 22.24$ & $100 \pm 0$ & $100 \pm 0$ & $100 \pm 0$ & $100 \pm 0$ \\
\cline { 2 - 7 } & S.P & $28.12 \pm 18.75$ & $100 \pm 0$ & $100 \pm 0$ & $100 \pm 0$ & $100 \pm 0$ \\
\cline { 2 - 7 } & S.RWA & $85.94 \pm 10.67$ & $100 \pm 0$ & $0 \pm 0$ & $0 \pm 0$ & $0 \pm 0$ \\
\hline Moyenne $L o c a l e$ & $65.62 \pm 28.32$ & $87.5 \pm 22.36$ & $75 \pm .72$ & $50 \pm 51.6$ & $50 \pm 51.64$ \\
\hline Effet traitement & 0.0000 & 0.000 & 0.000 & 0.000 & 0.000 \\
\hline Effet variété & 0.812 & 0.000 & 0.127 & 0.049 & 0.049 \\
\hline Interaction & 0.408 & 0.000 & 0.083 & 0.015 & 0.015 \\
\hline
\end{tabular}

(S.KAS : Sol de la ferme Kassapa, S.KI : Sol de Pengapenga, S.P, S.RWA : Sol de la Ruashi).

En outre, par rapport à l'utilisation des dites variétés, le test de la variance montre une différence significative entre les traitements $(p=0.000)$ seulement à 30 jours d'observation. C'est-à-dire la variété a influencée significativement le développement des feuilles à 30 jours après semis. Le meilleur taux en nombre des feuilles a été obtenu avec les sols de Kipushi et Kassapa, alors que les faibles pourcentages ont été obtenus avec le sol du quartier Pengapenga à 30 jours et Ruashi à 30 et à 45 jours.

Tableau 3 : Nombre des feuilles des plantes de Solanum macrocarpon 90 jours après semis

\begin{tabular}{|c|c|c|c|c|c|}
\hline Variété & Traitement & NBF 30 & NBF 45 & NBF 60 & NBF 90 \\
\hline \multirow{4}{*}{$\begin{array}{l}\text { Variété } \\
\text { Améliorée(Afrisem) }\end{array}$} & S.KAS & $3 \pm 0$ & $5.00 \pm 00$ & $10.00 \pm 1.1$ & $16.5 \pm 1.29$ \\
\hline & S.KIP & $4 \pm 0$ & $6.00 \pm 02$ & $10.25 \pm 0.9$ & $16.5 \pm 2.38$ \\
\hline & S.P & $2 \pm 0$ & $0.00 \pm 00$ & $0.0 \pm 0.00$ & $0.00 \pm 0.00$ \\
\hline & S.RWA & $3 \pm 0$ & $2.75 \pm 00$ & $0.0 \pm 0.00$ & $0.00 \pm 0.00$ \\
\hline \multicolumn{2}{|l|}{ Moyenne } & $3.00 \pm 0.73$ & $3.44 \pm 2.2$ & $5.06 \pm 5.27$ & $8.25 \pm 8.61$ \\
\hline \multirow[t]{4}{*}{ Variété Locale } & S.KAS & $2.75 \pm 0.0$ & $5.00 \pm 00$ & $10.00 \pm 1.6$ & $15.75 \pm 1.26$ \\
\hline & S.KIP & $3.00 \pm 0.0$ & $5.00 \pm 00$ & $10.25 \pm 1.5$ & $16.50 \pm 2.38$ \\
\hline & S.P & $1.00 \pm 1.5$ & $0.00 \pm 00$ & $0.0 \pm 0.00$ & $0.00 \pm 0.00$ \\
\hline & S.RWA & $2.75 \pm .50$ & $3.75 \pm 00$ & $0.0 \pm 0.00$ & $0.00 \pm 0.00$ \\
\hline \multicolumn{2}{|l|}{ Moyenne } & $2.38 \pm 1.02$ & $3.44 \pm 213$ & $5.06 \pm 5.32$ & $8.06 \pm 8.42$ \\
\hline \multicolumn{2}{|l|}{ Effet traitement } & 0.000 & 0.000 & 0.000 & 0.000 \\
\hline \multicolumn{2}{|l|}{ Effet variété } & 0.000 & 1.000 & 1.000 & 0.698 \\
\hline \multicolumn{2}{|l|}{ Interaction } & 0.207 & 0.000 & 1.000 & 0.926 \\
\hline
\end{tabular}

(S.KAS : Sol de la ferme Kassapa, S.KI : Sol de Pengapenga, S.P, S.RWA : Sol de la Rwashi). 

variétés (locale et améliorée) de légumes dans la région de Lubumbashi (RD. Congo).

Cependant, l'interaction entre traitement et la variété a eu des effets significatifs au $45^{\text {ème }}$ et au jour d'observations ; sauf à 30,60 et 90 jours où la différence réciproque a eu des effets non significatifs entre les traitements.

Tailles des plantes: Dans le tableau 4, concernât l'utilisation des types de sols, la meilleure hauteur de la plante a été obtenu avec le sol de Kipushi suivit de celui de Kassapa à toute les différentes dates d'observations, alors que les sol de Ruashi et le sol de Pengapenga ont présentés un taux faible en hauteur des plantes, contrairement à 30, 45, 60 et 90 jours; seuls le sol de Kipushi et Kassapa avait une montaison élevé contrairement aux sol de Pengapenga et Ruashi ont donnés une faible hauteur qu'au 30 et $45^{\mathrm{eme}}$ jour, alors

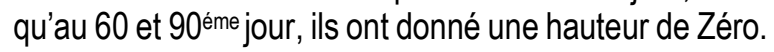

A ce qui concerne l'utilisation des dites variétés, le test de la variance révèle des différences significatives entre les traitements $(p=0.000)$ à toutes les dates d'observation; c'est-à-dire la variété a influencée significativement la survie ainsi que la hauteur des plantes à $30,45,60$ et 90 jours après semis ; le meilleur taux de croissance a été obtenu avec le sols des environs de Kipushi ensuite Kassapa, alors que la faible montaison a été obtenu avec le sol du quartier Pengapenga à 30 jours et Ruashi à 30 et à 45 jours. C'est ainsi que l'interaction entre traitement et la variété a eu des effets significatifs au 30 èmeet 45ème jours d'observations sauf à 60 et 90 jours où l'effet était non significatif entre les traitements.

Tableau 4 : Croissance des plantes de Solanum macrocarpon 90 jours après semis

\begin{tabular}{|c|c|c|c|c|c|}
\hline Variété & Traitement & HPL30 & HPL45 & HPL60 & HPL90 \\
\hline \multirow{4}{*}{$\begin{array}{l}\text { Variété } \\
\text { Améliorée(Afrisem) }\end{array}$} & S.KAS & $6.08 \pm 0.88$ & $8.30 \pm 2.14$ & $15.75 \pm 1.4$ & $26.75 \pm 7.04$ \\
\hline & S.KIP & $8.93 \pm 0.71$ & $1085 \pm 1.97$ & $15.50 \pm 1.2$ & $22.75 \pm 5.12$ \\
\hline & S.P & $1.15 \pm 0.24$ & $0.00 \pm 0.00$ & $0.00 \pm 0.00$ & $0.00 \pm 0.00$ \\
\hline & S.RWA & $2.38 \pm 0.22$ & $0.50 \pm 0.28$ & $0.00 \pm 0.00$ & $0.00 \pm 0.00$ \\
\hline \multicolumn{2}{|l|}{ Moyenne } & $4.63 \pm 3.22$ & $5.41 \pm 4.64$ & $7.81 \pm 8.12$ & $12.38 \pm 13.44$ \\
\hline \multirow[t]{4}{*}{ Variété Locale } & S.KAS & $3.08 \pm 1.73$ & $4.19 \pm 1.43$ & $14.50 \pm 2.6$ & $29.50 \pm 1.00$ \\
\hline & S.KIP & $4.15 \pm 0.82$ & $7.00 \pm 2.04$ & $17.25 \pm 4.4$ & $27.75 \pm 11.44$ \\
\hline & S.P & $0.13 \pm 0.15$ & $0.00 \pm 0.00$ & $0.00 \pm 0.00$ & $0.00 \pm 0.00$ \\
\hline & S.RWA & $1.77 \pm 0.59$ & $1.92 \pm 0.51$ & $0.00 \pm 0.00$ & $0.00 \pm 0.00$ \\
\hline \multicolumn{2}{|l|}{ Moyenne } & $2.28 \pm 1.79$ & $3.28 \pm 2.93$ & $7.94 \pm 8.58$ & $14.31 \pm 15.66$ \\
\hline \multicolumn{2}{|l|}{ Effet traitement } & 0.000 & 0.000 & 0.000 & 0.000 \\
\hline \multicolumn{2}{|l|}{ Effet variété } & 0.000 & 0.000 & 0.859 & 0.293 \\
\hline \multicolumn{2}{|l|}{ Interaction } & 0.000 & 0.000 & 0.512 & 0.719 \\
\hline
\end{tabular}

(S.KAS : Sol de la ferme Kassapa, S.KI : Sol de Pengapenga, S.P, S.RWA : Sol de la Rwashi).

Diamètre foliaire: Dans le tableau 5, par rapport à l'utilisation des types de sols, I meilleur diamètre de feuille a été obtenu avec le sol de Kipushi et Kassapa à toutes les différentes dates d'observations. Cependant, les sol de Ruashi et le sol de Pengapenga ont présentés un taux faible en diamètre foliaire, contrairement à 30, 45, 60 et 90 jours; seuls le sol de Kipushi et Kassapa avait présenté des surface foliaires élevé contrairement aux sol de Pengapenga qui a donné un faible taux de diamètre à 30 jours seulement, et sur le sol de Ruashi où la surface des feuilles plantes n'ont pu évoluer depuis 30 jusqu'au

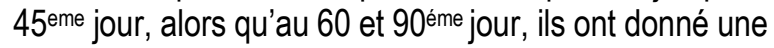
surface égale à Zéro. Ainsi donc, par rapport à l'utilisation des dites variétés, le test de la variance prouve des différences significatives entre les traitements $(p=0.001)$ à toutes les dates d'observation. C'est-à-dire la variété a influencée significativement le développement des feuilles à $30,45,60$ et 90 jours après semis ; le meilleur taux en diamètre a été obtenu avec les sols de la ferme Kassapa ensuite Kipushi, alors que les faibles diamètres ont été obtenu avec le sol du quartier Pengapenga à 30 jours et Ruashi à 30 et à 45 jours. C'est ainsi que l'interaction entre traitement et la variété a eu des effets significatifs au $30^{\mathrm{ème}}$ et au 90èmejour d'observations; sauf à 45 et 90 jours où la différence réciproque a eu des effets non significatif entre les traitements 
Tableau 5 : Diamètre au collet des plantes de Solanum macrocarpon 90 jours après semis

\begin{tabular}{|l|l|l|l|l|l|}
\hline Variété & Traitement & DF30 & DF45 & DF 60 & DF 90 \\
\hline Variété Améliorée(Afrisem) & S.KAS & $1.25 \pm 0.19$ & $3.95 \pm 0.60$ & $10.97 \pm 1.3$ & $11.88 \pm 1.93$ \\
\cline { 2 - 6 } & S.KIP & $0.55 \pm 0.40$ & $6.03 \pm 0.26$ & $6.03 \pm 1.26$ & $11.38 \pm 1.89$ \\
\cline { 2 - 6 } & S.P & $0.25 \pm 0.00$ & $0.00 \pm 0.00$ & $0.00 \pm 0.00$ & $0.00 \pm 0.00$ \\
\cline { 2 - 6 } & S.RWA & $0.50 \pm 0.00$ & $0.78 \pm 0.55$ & $0.00 \pm 0.00$ & $0.00 \pm 0.00$ \\
\hline Moyenne & S.KAS & $1.14 \pm 0.95$ & $2.69 \pm 2.60$ & $4.25 \pm 4.82$ & $5.81 \pm 6.13$ \\
\cline { 2 - 6 } Variété Locale & S.KIP & $1.60 \pm 0.57$ & $2.92 \pm 0.79$ & $10.28 \pm 0.9$ & $14.25 \pm 0.96$ \\
\cline { 2 - 6 } & S.P & $0.12 \pm 0.14$ & $0.12 \pm 1.04$ & $10.57 \pm 0.6$ & $12.62 \pm 1.25$ \\
\cline { 2 - 6 } & S.RWA & $0.48 \pm 0.10$ & $0.52 \pm 0.00$ & $0.00 \pm 0.00$ & $0.00 \pm 0.00$ \\
\hline Moyenne & $0.77 \pm 0.63$ & $1.89 \pm 1.85$ & $5.21 \pm 5.41$ & $6.72 \pm 7.00$ \\
\hline Effet traitement & 0.000 & 0.000 & 0.000 & 0.000 \\
\hline Effet variété & 0.000 & 0.000 & 0.001 & 0.029 \\
\hline Interaction & 0.000 & 0.051 & 0.000 & 0.121 \\
\hline
\end{tabular}

(S.KAS : Sol de la ferme Kassapa, S.KI : Sol de Pengapenga, S.P, S.RWA : Sol de la Rwashi).

Concentration de cuivre dans les biomasses de Solanum macrocarpon: Le tableau 6 présente les concentrations de cuivre dans les parties aériennes de l'aubergine. De ces résultats d'analyse de laboratoire, il ressort que les plantules d'aubergine n'ont pas présenté de risque de contamination cuprique. Les sols ayant présenté des teneurs élevées au seuil de toxicité n'ont présenté aucune survie jusqu'à la fin de l'expérimentation. Alors que le sol de Kassapa malgré ce léger dépassement de concentration (110 ppm) au-delà de la norme n'avait pas présenté de trace de contamination en cuivre.

Tableau 6 :Teneurs en Cuivre dans les parties aériennes de deux variétés d'aubergine (Solanum macrocarpon).

\begin{tabular}{|c|c|c|c|}
\hline Traitement & $\begin{array}{c}\text { Cu total } \\
\text { (ppm) }\end{array}$ & $\begin{array}{c}\text { Normes } \\
(\mathrm{ppm})\end{array}$ & Référence \\
\hline T1L & ND & \multirow{4}{*}{$\begin{array}{c}\text { Cu EDTA } \\
5-10\end{array}$} & \multirow{4}{*}{$\begin{array}{l}\text { Teneur maximale du règlement 1881/2006/CE (Tremel- } \\
\text { Schaub\&Feix, 2005). } \\
\text { Valeur normale (Kabata- Pendias\&Pendias, 2001) }\end{array}$} \\
\hline T3L & $\mathrm{ND}$ & & \\
\hline $\mathrm{T} 1 \mathrm{~A}$ & ND & & \\
\hline T2A & ND & & \\
\hline
\end{tabular}

T1L: sol de Kassapa avec variété améliorée locale. T1A: sol de Kassapa avec variété locale. T2A : sol de Pengapenga avec variété locale, T3L : sol Kipushi avec variété améliorée.

Taux de levée : Le taux de levée pour les deux variétés de la carotte est présenté dans la figure 1. Le résultat de l'analyse de la variance à deux facteurs montre que le sol a eu une influence significative sur les variétés $(P<0.05)$. La levée la plus grande a été observée sur le sol de
Kassapa pour les deux variétés (53 et $60 \%$ ) et sur les sols de Pengapenga aucune carotte n'avait poussée. II n'y a pas eu un effet d'interaction entre la source de provenance de sols et les variétés. 


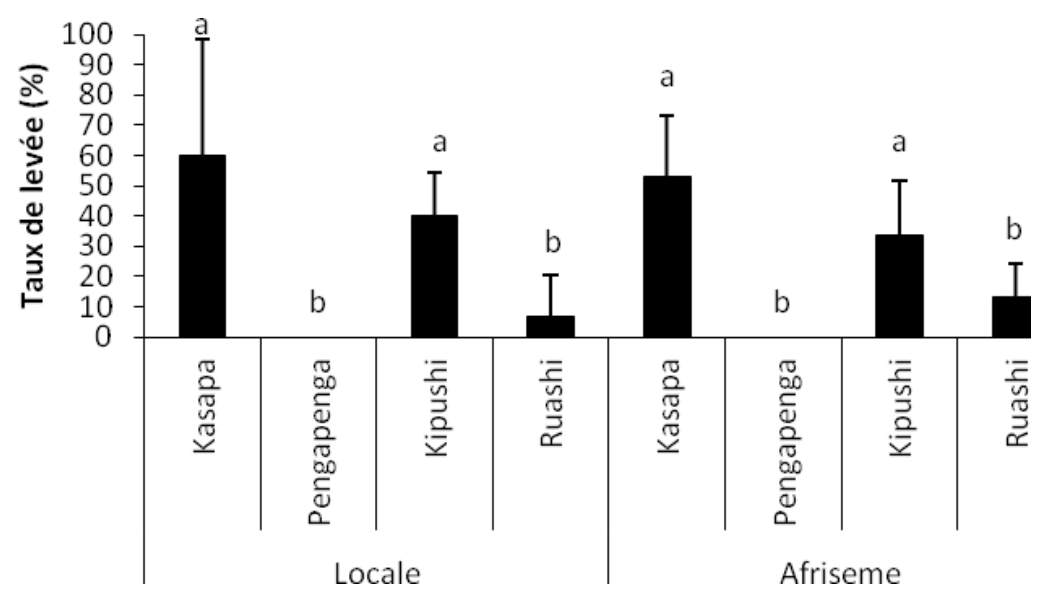

Figure 1 : Taux de levée de la carotte (variété locale et améliorée Afrisem).

Le taux de survie à 120 jours : Le taux de survie à 120 jours pour les deux variétés est présenté à la figure 2. Le résultat de l'analyse de la variance montre que le sol a influencé significativement la survie de deux variétés $(P<0.05)$. La survie la plus grande était observé sur le sol Kassapa et Ruashi (27\% pour Kassapa/variété locale et $27 \%$ pour Ruashi/variété Afrisem). La survie a été nulle sur les sols en provenance de Pengapenga et Ruashi pour la variété locale et seul Pengapenga pour la variété Afrisem.

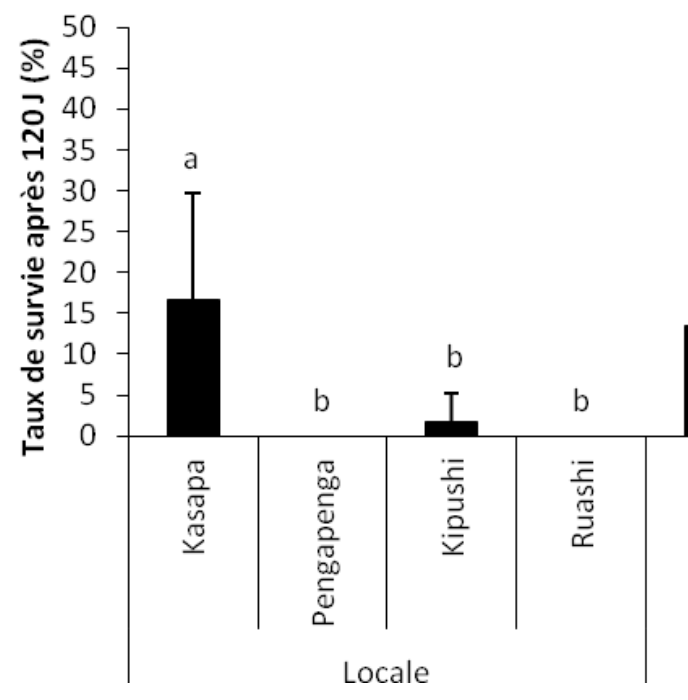

Figure 2 : Le taux de survie de la carotte pour les deux variétés (locale et Afrisem) à 120 jours

Tableau 7 : Concentration du cuivre dans les biomasses de la carotte

\begin{tabular}{|l|l|l|l|l|}
\hline Traitement & Variété & Cuivre total (ppm) & Norme $(\mathbf{p p m})$ & Référence \\
\hline T4n & Afrisem & 62 & 05 à 10 & Coppenet et al, 1993; Chevery, 1994 \\
\hline T3n & Afrisem & N.D & & \\
\hline T3m & Locale & ND & & \\
\hline T1m & locale & 39 & & \\
\hline T1n & Afrisem & 30 & & \\
\hline
\end{tabular}

T4n : sol de Pengapenga avec la variété améliorée ; T3n : sol de Kipushi avec la variété améliorée ; T3m : sol de Kipushi avec la variété locale ; T1m : sol de Kassapa avec la variété locale ; T1n : sol de Kassapa avec la variété améliorée, ND : non déterminé. 

variétés (locale et améliorée) de légumes dans la région de Lubumbashi (RD. Congo).

\section{DISCUSSION}

Concentration du cuivre dans les différents substrats potagers de la région de Lubumbashi : Des résultats obtenus des analyses de laboratoire, il ressort que les teneurs en cuivre dans les sols ont largement dépassés la norme de contamination fixée par l'OMS. Ces fortes teneurs en cuivre sont fonction des intenses activités minières dans la région (Macnair, 2003). Les études effectuées par Winterhalder (1996) cité par Linxian et al., 2013, dans la région de Sudbury avaient montrées que les activités minières (fonderie de Cu-Ni) avait conduit à la disparition de plus de 36000 hectares de forets naturelles et à la dénudation de plus de 10000 hectares de sol. Toutefois des situations similaires ont été observées par les études effectuées par Kikilä (2003) dans la région de Harjavalta (En Finlande) qui avait montré que les activités minières notamment la fonderie de cuivre et nickel était responsables de la dégradation de la diversité des espèces et du couvert végétal de pin sylvestre. La contamination de la chaine alimentaire est un problème réel dans la province du Haut-Katanga, les études réalisée par (Vande Weghe et al., 2005), nous renseignent que le bassin de la Lufira contient des teneurs très élevées en etms notamment (Cuivre, Zinc, Arsenic, Plomb, Cadmium et Cobalt). Le centre caryer confirme que la contamination dans la région de Lubumbashi est réelle, partant de ces résultats, il ressort que l'eau, le sol ainsi que les plantes liée à l'exploitation du cuivre et du cobalt par les entreprises Chemaf et Rwashi Mining à Tshiamilemba et Kabetsha présentés des teneurs largement supérieures au seuil de toxicité. Concentration du cuivre dans les biomasses de la carotte installée sur différents substrats : Les résultats de laboratoire obtenus montre que les deux variétés ont accumulés des fortes teneurs en cuivre sur les sol de Kassapa trois fois supérieures que la normale, alors que les plantes ayant poussées sur le sol de Rwashi ont présentés de teneurs six fois supérieures que la norme (seuil de toxicité) contrairement au sol de Kipushi qui n'avait présenter aucune trace de contamination cuprique, ces fortes teneurs dans les plantes de la carotte sont dues à des fortes teneurs retrouvées dans les sols. Nos résultats sont similaires ave ceux obtenus par Temgoua. E et al., 2015 dans la région de Dschang, qui ont trouvés que les plantes de carottes, d'amarantes installées sur substrat contaminés en cuivre, zinc, avait présentés des teneurs très élevées en ces ETMs excédant le seuil de toxicité fixé. Les études effectuées par (Islam et al., 2007 ; Bouchouata et al., 2011), ont montrés que les légumes cultivés sur des sols contaminés par les métaux lourds avaient accumulés des concentrations élevées pouvant constituer des risques de santé énormes chez les consommateurs. Des études similaires ont démontrées que les légumes prennent les métaux lourds en les absorbant des sols ou par exposition de différentes parties de la plante à l'air en provenance des sites pollués (Zurera-Cosano et al., 1989). Cependant, les études effectuées par Alegria et al., 1992, ont étudié les rapports entre la concentration du cobalt, du cuivre, et du zinc du sol et celles des légumes. De ces résultats, ils ont obtenu des corrélations significatives entre les concentrations totales et extractibles du cobalt du sol et celle des racines et des bulbes d'une part, et entre les concentrations totales du cuivre du sol et celles des inflorescences d'autre part.

Concentration du Cuivre dans les parties aériennes des plantes d'aubergine: L'analyse au laboratoire prouve que les plantules d'aubergines ayant poussée sur le substrat de Kipushi et Kassapa n'ont pas présentées des risques de contamination (Oppm) en cuivre; cette observation conduit à dire que les plantes installées sur les sols de Kipushi n'ont pas pu accumuler le cuivre, quel que soit la différence de variété. Cependant, nous avons observé que les plantes installées sur les sols de Pengapenga et Rwashi n'ont pas pu évoluer à partir du trentième jour après le semis, et les analyses du laboratoire indiquent des contaminations excessives sur les deux sols. Bien que la matière organique contribue généralement a l'immobilité du $\mathrm{Cu}$ en formant de fortes liaisons, elle peut aussi augmenter la solubilité du Cu par la formation de composes solubles (CCME, 1997). Le Cu a un minimum de solubilité entre $\mathrm{pH} 6$ et 8 . Aux pH inferieurs à 6 , il est majoritairement sous forme $\mathrm{Cu} 2+$, et au-dessus de 8 sous forme complexe avec la matière organique soluble (Barber, 1995). La concentration en solution de $\mathrm{Cu}$ augmente fortement pour des $\mathrm{pH}<4-5$ (OFEFP, 1996). Le Cu semble poser plus de problèmes de carence chez les végétaux, le bétail et l'homme, que de toxicité. Les manifestations pathologiques chez l'homme sont plutôt liées a une carence en $\mathrm{Cu}$ qui entraine anémie résistante au traitement par le $\mathrm{Fe}$, retards de croissance associes a des troubles du métabolisme osseux, lésions cardiaques, troubles du système nerveux. (Bourrelier et Berthelin, 1998) ; ceci pourrait être l'une des causes qui ont fait qu'il n'y ait pas une bonne croissance sur les substrats Pengapenga et Rwashi. Cependant, le $\mathrm{Cu}$ peut provoquer une phytotoxicité dans certains cas, comme sur des sols acides anciennement plantes en vignes ayant été traites a la bouillie bordelaise pendant des années. II induit alors des symptômes de la chlorose ferrique et une diminution 
de l'absorption du Fe et du P (Loue, 1986). Par contre, la teneur en cobalt de tous ces sols contaminés (Kassapa, Kipushi, Rwashi et Pengapenga) est non déterminée. Ce phénomène se justifierait par le fait qu'il existe de effets d'antagonismes entre éléments du sol, mais encore la carence en oxydes de $\mathrm{Mn}$ et $\mathrm{Fe}$, qui gouvernent sa présence et sa disponibilité; hormis l'acidité du sol (Moussavou, 2010). Temgoua E., et al., 2015, montrentle risque de contamination de la chaine alimentaire, partant de ces études effectuées au Cameroun sur la culture de

\section{CONCLUSION}

Cette étude avait pour objectif d'évaluer le comportement de deux variétés d'aubergines et de carottes installées sur les différents sols contaminés dans la région de Lubumbashi. Pour se faire, une certaine dose des amendements minéraux a été appliquée sur les sols Rwashi, Pengapenga, Kassapa et Kipushi contaminés en éléments trace métalliques. Deux essais ont été installés en vue d'évaluer les meilleures variétés adaptées à tolérer la contamination cuprique. Les expérimentations ont été conduites selon un dispositif en bloc complet randomisé comprenant 4 répétitions. Les traitements comprenaient quatre types de sol (Sol de Kipushi, sol de Kassapa, sol de Pengapenga et sol de Ruashi. Les résultats obtenus montrent d'une part qu'il n'y a pas

\section{RÉFÉRENCES BIBLIOGRAPHIQUES}

Adjia, R. 2010. Métaux lourds dans les légumes des jardins maraîchères de Ngaoundéré : niveau de toxicité et source de contamination, Thèse de Doctorat PhD, Université de Ngaoundéré. 212p.

Alegria, A., Barbera, R., Errecalde, F., Farre, R. and Largada, M.J. 1992. Relationship between cobalt, copper and zinc content of soil and vegetable. Nahrung 36 (5) : 451-460.

Bruneau J.C., 1983. Cartographie de l'environnement et aménagement urbain à Lubumbashi. Rev. Int. Écol. Géogr. Trop., 1(4), 19-47.

Bigdeli, M. and Seilsepour, M. 2008. Investigation of metal accumulation in some vegetable irrigated with waste water in Shahre Rey-Iran and Toxicological Implications. American- Eurasian Journal of Agric \& environ. Sci. 4 (1) : 86-92.

Bouchouata, O., Jaafar, B., Bounakhla, M., Doukkali, A. and Attarassi, B. 2011. Étude de la contamination par les métaux lourds des eaux d'irrigation et les cultures maraîchères dans la zone M'nasra (Gharb, Maroc), ScienceLib Editions Mersenne, 3, № 111006 ISSN 21114706. l'aubergine installés sur sol contaminés en ETMs, il ressort que les plantules récoltées avaient présenter des teneurs excédant la valeur seuil fixée par l'OMS, contrairement à nos résultats qui n'ont pas présenter des risques de contamination cuprique pour toutes les variétés. Par ailleurs, les travaux de (Bigdeli et Seilsepour, 2008; Adjia, 2010), ont montrés que la contamination des racines par les métaux lourds du sol est nettement supérieure à celle des parties aériennes.

risque de contamination de la chaine alimentaire, car l'analyse de laboratoire révèle que les biomasses d'aubergine n'ont pas présenté des teneurs en éléments traces pour le cuivre, et d'autre part que le risque de contamination existe eu égard aux résultats obtenus dans les biomasses de la carotte et dans les différents sols, les teneurs en cuivre ont dépassé les normes de contamination. Les suggestions que nous pouvons recommander aux futurs chercheurs intéressés sur la thématique de notre étude sont telles que :

- une étude sur terrain soit implantée dans le but de comparer la vigueur de croissance de ces deux variétés d'aubergines, installées sur sol contaminés.

Bourrelier P.H. \& Berthelin J., 1998. Contamination des sols par les éléments traces : les risques et leur gestion. Paris : Lavoisier Tec \& Doc.

Cabala J., Smieja-Krol B. Jablonska M.,Chrost L., 2012. Mineral components in a peat deposit: Looking for signs of early mining and smelting activities in Silesia-Cracow region (Southern Poland) FullText. Article. Aug 2012.

Cheverry C., 1994. La dégradation chimique des sols en Bretagne. Étude et Gestion des sols, $1: 7-21$

Coppenet M., Golven J., Simon J.-C., Le Corre L., Le Roy M., 1993. Évolution chimique des sols en exploitations d'élevage intensif : exemple du Finistère. Agronomie, 13: 77- $83 p$.

Clemente R., Walker D.J., Bernal M.P., 2005. Uptake of heavy metals and As by Brassica juncea grown in a contaminated soil in Aznacollar (Spain). The effect of soil amendements. Environmental Pollution 138, 46-58.

Cui Y., Wang Q., Christie P. 2004, Effect of elemental Sulphur on uptake of Cadmium, Zinc, and Sulphur by oilseed Rape growing in soil 
contaminated with Zinc and Cadmium. Soil Science and Plant Analysis., 35, 2905-2916.

CCME, 2008. Canadian soil quality guidelinesCarcinogenic and other polycyclic aromatic hydrocarbons (PAHs) (Environmental and Human Health Effects) - Scientific supporting Document. Canadian Council of Ministers of the Environment. 210 pages, www.ccme.cal assets/pdf/pah sogg ssd 1401.pdf.

FAO, 2005. Notions de nutrition des plantes et de fertilisation des sols. Manuel de formation, Projet Promotion de l'Utilisation des Intrants agricoles par les Organisations de Producteurs du Niger, Rome, FAO.

Gonzales, X.I., Aboal, J.R., Fernandez, J.A. and Carballeira, A. 2008. Heavy metal transfers between trophic compartments in different ecosystems in Galicia (northwest Spain): Essential elements. Archives of Environmental Contamination and Toxicology 55: 691-700.

Islamul, E., Yang, X., He, Z. and Mahmood, Q. 2007. Assessing potential dietary toxicity of heavy metal in selected vegetables and food crops. $J$. Zhejiang Univ. Sci. B. 8 (1) : 1-13.

Kitobo WS. (2009). Dépollution et valorisation des rejets miniers sulfurés du Katanga : Cas des taillings de l'ancien concentrateur de Kipushi. Thèse de doctorat, Faculté des Sciences Appliquées, Université de Liège. 254p.

Kabala C., Singh B.R., 2001. Fractionation and mobility of copper, lead and zinc in soil profiles in the vicinity of a copper smelter. J. Environ. Qual. 30: 485-492

Manda BK. (2009). Évaluation de la contamination de la chaine trophique par les métaux lourds dans le bassin de Lalufira supérieures (Katanga/RD Congo). Mémoire de DEA inédit. Faculté des Sciences Agronomiques/Université de Lubumbashi. 50p

Kasongo Lenge Mukonzo Emery, Mwamba Mulembo Théodore, Tshipoya Masumbuko Patient, Mukalay Muamba Joseph, Useni Sikuzani Yannick, Mazinga Kwey Michel, Nyembo Kimuni Luciens., 2013. Réponse de la culture de soja (Glycine max L. (Merril) à l'apport des biomasses vertes de Tithonia diversifolia (Hemsley) A. Gray comme fumure organique sur un Ferralsol à Lubumbashi, R.D. Congo. Journal of Applied Biosciences 63: 4727 - 4735 ISSN 1997-5902
Kiikkilä O. 2003. Heavy-metal pollution and remediation of forest soil around the Harjavalta $\mathrm{Cu}-\mathrm{Ni}$ smelter, in SW Finland. Silva Fennica. 37(3), 399- 415.

Kabata-Pendias A. 2001. Trace elements in soils and plants. CRC, LLC. Boca Raton, 413p.

Macnair MR. 2003. The Hyper accumulation of Metals by Plants. Advances in Botanical Research 40:65105

Mbenza M, Aloni K and Muteb M., 1989. Quelques considérations sur la pollution de l'air à Lubumbashi (Shaba, Zaïre). Géo-eco-trop, 13 (1-4), 113-125

Malaisse, F., 1997. Se nourrir en forêt Claire Africaine. Approche écologique et nutritionnelle. Presses agronomique de Gembloux. p. 15-35.

Mpundu M.M., 2010. Contaminations des sols en Éléments Traces Métalliques à Lubumbashi (Katanga/RD Congo). Évaluation des risques de contamination de la chaîne alimentaire et choix de solutions de remédiation. Thèse de doctorat: Faculté des Sciences Agronomiques, Université de Lubumbashi (RD Congo).

Mpundu M. M., Useni S. Y., Mwamba M. T., Kateta M. G., Mwansa M., llunga K., Kamengwa K. C., K. K., Nyembo K. L., 2013b. Teneurs en éléments traces métalliques dans les sols de différents jardins potagers de la ville minière de Lubumbashi et risques de contamination des cultures potagères. Journal of Applied Biosciences 65:4957-4968

Mpundu M. M, Useni S. Y, Nyembo K. L, Colinet G., 2014. Effets d'amendements carbonatés et organiques sur la culture de deux légumes sur sol contaminé à Lubumbashi (RD Congo). Biotechnol. Agron. Soc. Environ. 18(2), 1-9

Mougeot et Moustier., 2004. Les dynamiques de l'agriculture urbaine: caractérisation et évaluation In : Smith.O, Moustier, P., Mougeot, L. et Fall, A. Développement durable de l'agriculture urbaine en Afrique francophone. Enjeux, concepts et méthodes, CIRAD et CRDI. pp. 23-29.

Mc Lean, C.M., Koller, C.E., Rodger, J.C. and MacFarlane, G. R. 2009. Mammalian hair as an accumulative bioindicator of metal bioavailability in Australian terrestrial environments. Science of the Total Environnement 407 (11): 3588-3596.

Mulaji K.C., 2010. Utilisation des composts de biodéchets ménagers pour l'amélioration de la fertilité des sols acides de la province de Kinshasa 
(République Démocratique du Congo). Thèse de doctorat, université de Liège- Gembloux AgroBiotech, 220p.

Munyemba K. F. 2010. Quantification et modélisation de la dynamique paysagère dans la région de Lubumbashi: évaluation de l'impact écologique des dépositions issues de la pyrométallurgie. Thèse Doctorale, Université de Lubumbashi ; Sciences Agronomiques, 2010

Ngoy S.M., 2010. Phytostabilisation des sols contaminés en métaux lourds par l'activité minière au Katanga « Cas du quartier Gécamines/Penga Penga contaminés en métaux par les émissions de la fonderie de cuivre de l'Usine Gécamines/Lubumbashi ». Thèse de doctorat. Université de Lubumbashi. $10 \mathrm{p}$.

Sylvain Boisson, Julien Collignon, Serge Langunu, Julie Lebrun, Mylor Ngoy Shutcha, Grégory Mahy. 2006. Concilier la phytostabilisation des sols pollués avec la conservation de la flore cuprocobalticole dans la région de Lubumbashi : une stratégie nouvelle pour valoriser les écosystèmes extrêmes.

Remona E., J.-L. Bouchardonb, B. Corniera, B. Guyb, J.C. Leclerca, O. Faurea, 2005. Soil characteristics, heavy metal availability and vegetation recovery at a former metallurgical landfill : Implications in risk assessment and site restoration. Environmental Pollution 137 :316323

Shutcha M.N. et al., 2010. Phytostabilisation of coppercontaminated soil in Katanga : an experiment with three native grasses and two amendements. Int. J. Phytoremediation, 12(6), 616-632.

Tremel-Shaub A., Feix I., 2005. Contamination des sols vers les plantes. EDP Sciences ADEME 156p

Tembo DB, Sichilongo K, Cernak J 2006. Distribution of copper, lead, cadmium and zinc concentrations in soils around Kabwe Town in Zambia. Chemosphere, 63: 497-501.

Temgoua, E., Pfeifer, H.R. and Bitom, D. 2003. Trace element differentiation in ferruginous slope accumulation patterns of southern Cameroon, the role of climate change. Science of The Total Environment 303(3): 203 - 214.

Temgoua. E, H. Ntangmo Tsafack1, H.-R. Pfeifer and T. Njine., 2015. Teneurs en éléments majeurs et oligoéléments dans un sol et quelques cultures maraîchères de la ville de Dschang, Cameroun
African Crop Science Journal, Vol. 23, No. 1, pp. $35-44$.

Vande Weghe J. P., Franssen J., Kalambay G., Kramkimel J.D. \& Musibono D. 2005. Étude "Profil environnemental (PEP) de la République Démocratique du Congo"; Délégation de la commission Européenne. EURATA, $228 \mathrm{p}$.

Zurera-Cosano, G., Moreno-Rojas, R., Salmeron-Egea, J. and Pozo Lora, R., 1989. Heavy metal uptake from greenhouse border soils for edible vegetable. Journal of the Science of Food and Agriculture 49(3): 307-314. 